\title{
Hematological Indexes Can Be Used to Predict the Incidence of Hypothyroidism in Nasopharyngeal Carcinoma Patients after Radiotherapy
}

\author{
Ling Zhou, ${ }^{1,2,3,4}$ Jia Chen, ${ }^{5}$ Chang-Juan Tao, ${ }^{1,2,3}$ Shuang Huang, ${ }^{1,2,3}$ Jiang Zhang, ${ }^{1,2,3}$ \\ Wei Shen, ${ }^{5}$ Chao-Nan Zhu, ${ }^{5}$ Ming Chen $\mathbb{1}^{1,2,3}$ Zhong-Hua Yu, ${ }^{6}$ and Yuan-Yuan Chen $\mathbb{1}^{1,2,3}$ \\ ${ }^{1}$ Institute of Cancer and Basic Medical (ICBM), Chinese Academy of Sciences, Hangzhou, China \\ ${ }^{2}$ Department of Radiation Oncology, Cancer Hospital of University of Chinese Academy of Sciences, Hangzhou, China \\ ${ }^{3}$ Department of Radiation Oncology, Zhejiang Cancer Hospital, Hangzhou, China \\ ${ }^{4}$ Postgraduate Education, Guangdong Medical University, Zhanjiang, China \\ ${ }^{5}$ Hangzhou YITU Healthcare Technology Co., Ltd., Hangzhou, China \\ ${ }^{6}$ Department of Oncology, The Affiliated Hospital of Guangdong Medical University, Zhanjiang, China
}

Correspondence should be addressed to Yuan-Yuan Chen; chenyy@zjcc.org.cn

Received 21 January 2020; Revised 8 April 2020; Accepted 22 April 2020; Published 13 May 2020

Academic Editor: Chi-Un Pae

Copyright (c) 2020 Ling Zhou et al. This is an open access article distributed under the Creative Commons Attribution License, which permits unrestricted use, distribution, and reproduction in any medium, provided the original work is properly cited.

\begin{abstract}
Background. This study explored the relationship between thyroid-associated antibodies, immune cells, and hypothyroidism to establish a predictive model for the incidence of hypothyroidism in patients with nasopharyngeal carcinoma (NPC) after radiotherapy. Methods. A total of 170 patients with NPC treated at the Cancer Hospital of University of Chinese Academy of Sciences between January 2015 and August 2018 were included. The complete blood count, biochemical, coagulation function, immune cells, and thyroid-associated antibodies tested before radiotherapy were evaluated. A logistic regression model was performed to elucidate which hematological indexes were related to hypothyroidism development. A predictive model for the incidence of hypothyroidism was established. Internal verification of the multifactor model was performed using the tenfold cross-validation method. Results. The univariate analysis showed that immune cells had no statistically significant differences among the patients with and without hypothyroidism. Sex, N-stage, antithyroid peroxidase antibody (TPO-Ab), antithyroglobulin antibody (TG-Ab), thyroglobulin (TG), and fibrinogen (Fb) were associated with hypothyroidism. Males and early N-stage were protective factors of thyroid function, whereas increases in TPO-Ab, TG-Ab, TG, and Fb counts were associated with an increased rate of hypothyroidism incidence. The multivariate analysis showed that TPO-Ab, TG-Ab, TG, and $\mathrm{Fb}$ were independent predictors of hypothyroidism. The comprehensive effect of the significant model, including TPO-Ab, TG$\mathrm{Ab}, \mathrm{TG}$, and $\mathrm{Fb}$ counts, represented the optimal method of predicting the incidence of radiation-induced hypothyroidism $(A U C=0.796)$. Tenfold cross-validation methods were applied for internal validation. The AUCs of the training and testing sets were 0.792 and 0.798 , respectively. Conclusion. A model combining TPO-Ab, TG-Ab, TG, and Fb can be used to screen populations at a high risk of developing hypothyroidism after radiotherapy.
\end{abstract}

\section{Background}

Nasopharyngeal carcinoma (NPC) is one of the common malignant tumors in Southern China and Southeast Asia. Moreover, NPC is associated with previous Epstein-Barr virus (EBV) infection, for which radiotherapy is the primary treatment. Due to the development of intensity-modulated radiation therapy (IMRT) and comprehensive therapy, the five-year survival rate can be as high as $80 \%[1,2]$. In addition, with the extension of survival time, patient quality of life has been an issue of increasing attention. IMRT can increase the dose of radiation to the tumor target area and reduce the incidence of side effects in the surrounding normal tissues [3]. However, due to their special anatomical structure, the 
pituitary and thyroid glands will inevitably be exposed to a dose of radiation, which results in radiation-induced hypothyroidism. Hypothyroidism is a pathological condition resulting from thyroid hormone deficiency, which is divided into clinical and subclinical hypothyroidism. The incidence of hypothyroidism after radiotherapy increased from $20 \%$ to $60 \%$ during the era of IMRT treatment and could not be effectively controlled $[4,5]$. The most common symptoms of hypothyroidism consisted of fatigue, drowsiness, fear of cold, weight gain, constipation, and dry skin. In severe cases, it can even lead to heart disease, including coronary heart disease, heart failure, and other conditions [6]. Therefore, even during IMRT treatment, greater attention should be paid regarding the side effects on the thyroid glands after radiotherapy.

Recently, a large number of studies have reported that the immune system plays a key role in radiation response $[7,8]$, which is divided into adaptive and innate immunity. Adaptive immunity consists primarily of B and T lymphocytes. B lymphocytes are the precursors of plasma cells and are regulated by $\mathrm{T}$ lymphocyte subsets. T lymphocyte subsets are one of the most important cell groups in the immune system and can be divided into CD4+ and CD8+ T cell populations. Natural killer $(\mathrm{NK})$ cells are a type of innate immune cell that can activate the adaptive immune system via critical signals. Thus, T lymphocytes, B lymphocytes, and NK cells are the key mediators of the radiation-induced immune response. Moreover, evidence from other studies has demonstrated reduced efficacy for radiotherapy of patients who aredeficient in immune cells $[9,10]$. Currently, although the mechanism of radiation-induced hypothyroidism remains unclear, it is generally believed that radiation-induced immune response is one of the main mechanisms $[11,12]$. Some previous studies have demonstrated that the concentration of thyroidassociated antibodies (i.e., antithyroid peroxidase antibody (TPO-Ab) and antithyroglobulin antibody (TG-Ab)) can be correlated with hypothyroidism [13]. Moreover, the incidence of hypothyroidism is higher in patients positively expressing TPO-Ab and TG-Ab [14]. However, no relevant prediction model based on immune indicators has been established to predict the incidence of radiation-induced hypothyroidism in NPC. Therefore, this study is aimed at exploring the relationship between thyroid-associated antibodies, immune cells, and the incidence rate of hypothyroidism. The purpose of such findings was to explain the internal relationship between radiation-induced hypothyroidism and immune function, as well as establish a model with the relevant hematological indexes to predict the incidence rate of hypothyroidism in patients with NPC after radiotherapy.

\section{Methods}

2.1. Patient Selection. Patients with NPC treated at the Cancer Hospital of University of Chinese Academy of Sciences from January 2015 to August 2018 were included in the present study. The eligibility criteria for this study included patients with NPC diagnosed by pathology, who had complete medical records, and thyroid-associated antibody and immune function tests prior to radiotherapy. The follow-up period for the thyroid function test was greater than one year for all of the patients. Patients with immune system, hypothalamus, pituitary, or thyroid diseases were excluded from this study.

\subsection{Radiotherapy and Chemotherapy}

2.2.1. Chemotherapy. All patients received 2-3 cycles TP (docetaxel/paclitaxel+cisplatin/nedaplatin), PF (cisplatin/nedaplatin +fluorouracil), or TPF (docetaxel/paclitaxel+cisplatin/nedaplatin + fluorouracil)-induced chemotherapy. Chemotherapy with cisplatin or nedaplatin was performed simultaneously during radiotherapy.

2.2.2. Radiotherapy. All of the patients received IMRT. The specific doses assigned to the gross primary tumor, positive lymph nodes, high-risk areas, and low-risk areas were $66 \mathrm{~Gy}-74 \mathrm{~Gy}, 60 \mathrm{~Gy}-70 \mathrm{~Gy}, 60 \mathrm{~Gy}-62 \mathrm{~Gy}$, and $50 \mathrm{~Gy}-56 \mathrm{~Gy}$, respectively, which were divided into 33 fractions. The high-risk areas included the primary nasopharyngeal tumor and mucosa, and the low-risk areas included the posterior maxillary sinus, pterygopalatine fossa, parapharyngeal space, skull base, part of posterior ethmoid sinus, and bilateral lymphatic drainage area. The limit of endangering organs was subjected to the established guidelines [15].

2.3. Hematological Indexes. The complete blood count, biochemical, coagulation function, immune cell, and thyroidassociated antibody tests before radiotherapy were evaluated for all of the patients. The complete blood count included the white blood cells, neutrophils, lymphocytes, monocytes, eosinophils, basophils, red blood cells, platelets, and hemoglobin. Biochemical tests included the albumin, globulin, total protein, lipoprotein, alanine aminotransferase, aspartate aminotransferase, cholinesterase, creatinine, bilirubin, bile acid, uric acid, and glucose. The coagulation function test included the partial thromboplastin time, prothrombin time, and fibrinogen $(\mathrm{Fb})$ content. The immune cells included $\mathrm{T}$ cells, B cells, and NK cells. The thyroid-associated antibodies included TPO-Ab, TG-Ab, and thyroglobulin (TG).

2.4. Examination of Thyroid Function. All patients underwent a thyroid function test before and after radiotherapy, which included thyroid-stimulating hormone (TSH), triiodothyronine $\left(\mathrm{FT}_{3}\right)$, and free thyroxine $\left(\mathrm{FT}_{4}\right)$ using the electrochemiluminescence method with the SIEMENS ADVIA Centaur XP, with a reference range of TSH and $\mathrm{FT}_{4}$ being 0.380-4.340 IU/mL and 0.81-1.89 ng/dL, respectively. Biochemical hypothyroidism was defined as TSH concentrations above the reference range and $\mathrm{FT}_{4}$ concentrations within or lower than the normal range [12]. The above definitions cover both clinical and subclinical hypothyroidism. Central hypothyroidism was defined as low FT4 levels with low or low-to-normal levels of TSH. After radiotherapy, thyroid function tests were performed at least once every six months.

2.5. Statistical Analysis. Firstly, the clinical characteristics stratified by hypothyroidism were described using the means and standard deviations (for normally distributed variables), interquartile range (median (Q25-Q75)) (for abnormally 
distributed variables), or frequency and percentages (for categorical variables). Their differences were then correspondingly compared with a $t$-test, Wilcoxon rank sum test (or Mann-Whitney $U$ test), and Chi-square test (or Fisher's exact probability), respectively. Secondly, a series of univariate logistic regression models were performed to examine which of the clinical and immunological indexes were related to the development of hypothyroidism. Only the characteristics that were significantly different in the univariate analysis were included in the logistic regression model, and the independent predictors of hypothyroidism were identified using backward elimination $(P>0.1$ was excluded). Moreover, the internal verification of the multifactor model was performed using the tenfold cross-validation method. Thirdly, the cut-off for the immunological index was calculated using a receiver operating characteristics (ROC) curve. Finally, we established a joint prediction model according to the cut-offs of the significantly independent variables. $P<0.05$ was considered to be statistically significant. All analyses were performed using R-3.6.0.

\section{Results}

3.1. Patient Characteristics. The median follow-up time of the 170 patients was 20 months (13-36 months) and the incidence rate of primary hypothyroidism was $45.29 \%$ (77/170); no patients were diagnosed with central hypothyroidism. The average age of the patients was $51.55 \pm 10.55$ years old, including 115 males and 55 females. The patients' general clinical characteristics and the distribution of immune cells are listed in Table 1.

\subsection{Factors Related to Radiation-Induced Hypothyroidism.} The univariate analysis demonstrated that sex, N-stage, and pretreatment TPO-Ab, TG-Ab, TG, and $\mathrm{Fb}$ counts were associated with hypothyroidism. Among these factors, males and early $\mathrm{N}$-stage were protective factors of thyroid function, whereas the higher the pretreatment TPO-Ab, TG-Ab, TG, and $\mathrm{Fb}$ counts, the higher the hypothyroidism incidence rate. No differences were shown for the factors of age, clinical stage, chemotherapy, and other hematological indicators among the patients with and without hypothyroidism. The multivariate analysis showed that the pretreatment TPO$\mathrm{Ab}, \mathrm{TG}-\mathrm{Ab}, \mathrm{TG}$, and $\mathrm{Fb}$ counts were independent predictors of hypothyroidism (Table 2).

The cut-off for the immunological index was calculated using the ROC, and the optimal TPO-Ab, TG-Ab, TG, and $\mathrm{Fb}$ counts were determined using the maximum Youden index (specificity + sensitivity -1$)$. Patients were then accordingly reclassed into two groups with respect to the specific cut-off value; the critical values of TPO-Ab, TG-Ab, TG, and $\mathrm{Fb}$ related to the incidence of radiation-induced hypothyroidism were $7.40 \mathrm{IU} / \mathrm{mL}$ (normal value: 0-34 IU/mL), $57.40 \mathrm{IU} / \mathrm{mL}$ (normal value: $0-115 \mathrm{IU} / \mathrm{mL}$ ), $16.65 \mathrm{ng} / \mathrm{mL}$ (normal value: $1.40-78 \mathrm{ng} / \mathrm{mL}$ ), and $3.50 \mathrm{~g} / \mathrm{L}$ (normal value: 2-4 g/L), respectively. The incidence rates of hypothyroidism were $11.11 \%$ vs. $51.75 \%, 37.21 \%$ vs. $70.73 \%, 37.88 \%$ vs. $71.05 \%$, and $36.45 \%$ vs. $60.32 \%$, respectively (Table 3 ).
3.3. Prediction Model. The prediction models were built according to the results of the univariate and multivariate analyses. A full prediction model was established by including the indexes that were statistically significant in the univariate analysis, including sex, $\mathrm{N}$-stage, and TPO-Ab, TG$\mathrm{Ab}, \mathrm{TG}$, and $\mathrm{Fb}$ counts. A significant model was built by including indexes with significant statistical significance in the multivariate analysis, including TPO-Ab, TG-Ab, TG, and $\mathrm{Fb}$ counts (Table 4 ).

The ROC curves showed that the effect of combining TPO-Ab, TG-Ab, TG, and Fb with the four indexes to predict the incidence of hypothyroidism was significantly higher than that of their independent prediction (AUC $=0.796$ ). The combination of all relevant factors was used to establish a full model to predict the incidence of hypothyroidism $(\mathrm{AUC}=0.797)$ (Figure 1).

Furthermore, a tenfold cross-validation method was used to verify the multifactor model built with the full model and significant model. The AUCs of the training set for the full model and the significant model were 0.794 and 0.792 , respectively, and the AUCs of the testing set of the full model and the significant model were 0.774 and 0.798 , respectively (Figure 2). Therefore, the comprehensive effect of the significant model represented the best method of predicting the incidence of radiation-induced hypothyroidism, which showed $79.2 \%$ sensitivity and $67.7 \%$ specificity.

\section{Discussion}

Hypothyroidism is a common complication of nasopharyngeal carcinoma after radiotherapy. In our study, the incidence of biochemical hypothyroidism was $45.29 \%$, and no patients were diagnosed with central hypothyroidism, which may be due to insufficient follow-up time. Bhandare et al. showed that the incidence of central hypothyroidism was $5.4 \%$, and the median latency period was 4.8 years [16].

The thyroid gland is the largest endocrine gland in the body, which primarily secretes thyroid hormones and its insufficient secretion causes hypothyroidism. The clinical manifestations of hypothyroidism vary according to age and sex, with mild cases being asymptomatic, severe cases being life-threatening, and a mortality rate as high as $40 \%$ [17]. Currently, the pathogenesis of hypothyroidism remains poorly understood. Studies have shown that hypothyroidism is closely related to abnormal lipid metabolism, cardiovascular, autoimmune, and other diseases $[18,19]$.

It is now believed that radiation-induced hypothyroidism in patients with NPC is significantly related to the radiation dose, age, sex, and clinical stage. Sommat et al. [20] considered that a younger age and larger $\mathrm{T}$-stage were risk factors of hypothyroidism, whereas a thyroid $\mathrm{V}_{40} \leq 85 \%$ was a protective factor for thyroid function. Moreover, the retrospective analysis performed by Ling et al. [21] showed that thyroid $\mathrm{V}_{50}$ was an important predictor of radiationinduced hypothyroidism, and thyroid $\mathrm{V}_{50}<50 \%$ was closely related to the incidence of hypothyroidism. In addition, Zhai et al. [22] recommended that the thyroid dose be set to $V_{50} \leq 35 \%$ and $V_{45} \leq 50 \%$ to protect thyroid function. Since there is no unified standard for the optimal 
TABLE 1: Distribution of general clinical features and immunological indexes in 170 patients with NPC after IMRT.

\begin{tabular}{|c|c|c|c|c|}
\hline \multirow{2}{*}{ Variables } & \multicolumn{2}{|c|}{170 patients with NPC after IMRT } & \multirow{2}{*}{ Statistic } & \multirow{2}{*}{$P$ value } \\
\hline & Without hypothyroidism $(N=93)$ & With hypothyroidism $(N=77)$ & & \\
\hline Age (mean (SD)) & $52.66(10.84)$ & $50.22(10.1)$ & $t=1.50$ & 0.1347 \\
\hline Sex & & & $\chi^{2}=5.4499$ & $0.0196^{*}$ \\
\hline Male & $70(41.18)$ & $45(26.47)$ & & \\
\hline Female & $23(13.53)$ & $32(18.82)$ & & \\
\hline T-stage (2010UICC) & & & $\chi^{2}=0.6396$ & 0.4239 \\
\hline $\mathrm{T}_{1-2}$ & $16(9.41)$ & $17(10)$ & & \\
\hline $\mathrm{T}_{3-4}$ & $77(45.29)$ & $60(35.29)$ & & \\
\hline N-stage (2010UICC) & & & $\chi^{2}=4.5349$ & $0.0332^{*}$ \\
\hline $\mathrm{N}_{0-1}$ & $40(23.53)$ & $21(12.35)$ & & \\
\hline $\mathrm{N}_{2-3}$ & $53(31.18)$ & $56(32.94)$ & & \\
\hline M-stage (2010UICC) & & & & $0.3025^{\mathrm{a}}$ \\
\hline $\mathrm{M}_{0}$ & $90(52.94)$ & $71(41.76)$ & & \\
\hline $\mathrm{M}_{1}$ & $3(1.76)$ & $6(3.53)$ & & \\
\hline Clinical stage (2010UICC) & & & $\chi^{2}=2.0053$ & 0.1567 \\
\hline I-III & $43(25.29)$ & $44(25.88)$ & & \\
\hline IV & $50(29.41)$ & $33(19.41)$ & & \\
\hline Neoadjuvant chemotherapy & & & & $0.5141^{\mathrm{a}}$ \\
\hline No & $6(3.53)$ & $3(1.76)$ & & \\
\hline Yes & $87(51.18)$ & $74(43.53)$ & & \\
\hline BMI & & & & $0.4927^{\mathrm{a}}$ \\
\hline$<18.5$ & $6(3.53)$ & $2(1.18)$ & & \\
\hline$\sim 24.9$ & $64(37.65)$ & $53(31.18)$ & & \\
\hline$\geq 25$ & $23(13.53)$ & $22(12.94)$ & & \\
\hline $\mathrm{TPO}-\mathrm{Ab}$ & $12.5(7.4-17.3)$ & $14.6(11.1-28.1)$ & $Z=3.1572$ & $0.0016^{* *}$ \\
\hline TG-Ab & $11.2(10.2-16)$ & $14.1(10.1-151.2)$ & $Z=1.7728$ & 0.0763 \\
\hline TG & $7.5(4.56-14.18)$ & $11.95(4.8-22.36)$ & $Z=2.1271$ & $0.0334^{*}$ \\
\hline CD19+ & $7.8(4.8-10.9)$ & $7.8(5.7-11)$ & $Z=0.5964$ & 0.5509 \\
\hline $\mathrm{CD} 3+$ & $69.8(60-77.5)$ & $69.6(57.48-77.6)$ & $Z=-0.6934$ & 0.4881 \\
\hline $\mathrm{CD} 4+$ & $36.8(28.9-44.3)$ & $37.27(30.1-42.5)$ & $Z=0.1315$ & 0.8954 \\
\hline CD8+ & $26.13(20.6-33.9)$ & $25.2(19.8-32.4)$ & $Z=-0.8061$ & 0.4202 \\
\hline $\mathrm{CD} 4+/ \mathrm{CD} 8+$ & $1.4(0.9-1.9)$ & $1.4(1.1-2.1)$ & $Z=0.7255$ & 0.4681 \\
\hline CD38+ & $7(4.5-9.4)$ & $7.02(4.7-9.32)$ & $Z=0.2129$ & 0.8314 \\
\hline CD45RA- & $10.2(7.1-14.1)$ & $9.4(7-13.9)$ & $Z=-0.4383$ & 0.6612 \\
\hline CD45RA+ & $22(17.67-25.6)$ & $23.3(17.15-28.33)$ & $Z=0.8437$ & 0.3989 \\
\hline $\mathrm{CD} 45 \mathrm{RO}+$ & $22.1(16.6-25.7)$ & $22.13(17.2-27.9)$ & $Z=1.1489$ & 0.2506 \\
\hline CD45RA+/CD45RO+ & $2.7(1.9-3.8)$ & $2.95(1.9-3.9)$ & $Z=0.4885$ & 0.6252 \\
\hline CD56+ & $18.45(12.3-27.5)$ & $20.3(12.8-27.17)$ & $Z=0.659$ & 0.5099 \\
\hline NET cell & $2.8(1.8-5)$ & $3.2(1.6-4.6)$ & $Z=-0.1127$ & 0.9103 \\
\hline Neutrophils/lymphocytes & $2.35(1.55-4)$ & $3(1.42-5.3)$ & $Z=0.9251$ & 0.3549 \\
\hline EBV DNA & $0(0-500.99)$ & $0(0-737.08)$ & $Z=-0.0033$ & 0.9974 \\
\hline Cholinesterase & $7508(6620-8730)$ & 7737 (6776-9406) & $Z=1.3211$ & 0.1865 \\
\hline $\mathrm{Fb}$ & $3.17(2.58-3.54)$ & $3.5(3.03-3.91)$ & $Z=2.8379$ & $0.0045^{*}$ \\
\hline
\end{tabular}

Note: the values in the table are $N(\%)$ or median $\left(\mathrm{Q}_{25}-\mathrm{Q}_{75}\right)$ if there is no special marking; ${ }^{\mathrm{a}} \mathrm{P}$ value is calculated by Fisher's exact probability. ${ }^{*} P<0.05$; ${ }^{* *} P<0.01$; and ${ }^{* * *} P<0.001$. 
TABLE 2: Logistic regression analysis of radiation-induced hypothyroidism in 170 patients with NPC.

\begin{tabular}{|c|c|c|c|c|}
\hline \multirow{2}{*}{ Variables (reference group) } & \multicolumn{2}{|c|}{ Univariate analysis } & \multicolumn{2}{|c|}{ Multivariate analysis } \\
\hline & OR $(95 \% \mathrm{CI})$ & $P$ value & OR (95\% CI) & $P$ value \\
\hline Age & $0.98(0.95-1.01)$ & 0.1354 & & \\
\hline Sex male (versus female) & $0.46(0.24-0.89)$ & $0.0206^{*}$ & $0.73(0.33-1.61)$ & 0.4342 \\
\hline BMI underweight (versus normal) & $0.4(0.08-2.08)$ & 0.2396 & & \\
\hline BMI overweight (versus normal) & $1.16(0.58-2.3)$ & 0.2438 & & \\
\hline $\mathrm{T}$-stage $\mathrm{T}_{2-3}\left(\right.$ versus $\left.\mathrm{T}_{0-1}\right)$ & $0.73(0.34-1.57)$ & 0.4248 & & \\
\hline $\mathrm{N}$-stage $\mathrm{N}_{2-3}$ (versus $\mathrm{N}_{0-1}$ ) & $2.01(1.05-3.85)$ & $0.0344^{*}$ & $1.34(0.63-2.85)$ & 0.4527 \\
\hline$M$-stage $M_{1}$ (versus $M_{0}$ ) & $2.54(0.61-10.49)$ & 0.1993 & & \\
\hline Clinical stage IV (versus I-III) & $0.65(0.35-1.19)$ & 0.1576 & & \\
\hline Neoadjuvant chemotherapy (yes (versus no)) & $1.7(0.41-7.04)$ & 0.4635 & & \\
\hline TPO-Ab & $1.014(1.003-1.026)$ & $0.0144^{*}$ & $1.01(1.002-1.03)$ & $0.025^{*}$ \\
\hline TG-Ab & $1.004(1.001-1.006)$ & $0.0024^{* *}$ & $1.004(1.001-1.01)$ & $0.0112^{*}$ \\
\hline TG & $1.04(1.01-1.07)$ & $0.0105^{*}$ & $1.06(1.02-1.1)$ & $0.0009^{* * *}$ \\
\hline CD19+ & $1.01(0.94-1.09)$ & 0.7506 & & \\
\hline CD3+ & $0.99(0.97-1.02)$ & 0.4777 & & \\
\hline CD4+ & $1.00(0.97-1.03)$ & 0.9681 & & \\
\hline CD8+ & $0.98(0.95-1.02)$ & 0.3422 & & \\
\hline $\mathrm{CD} 4+/ \mathrm{CD} 8+$ & $1.16(0.82-1.63)$ & 0.4024 & & \\
\hline CD38+ & $0.99(0.92-1.06)$ & 0.7707 & & \\
\hline CD45RA- & $1.02(0.97-1.06)$ & 0.5055 & & \\
\hline CD45RA+ & $1.00(0.95-1.06)$ & 0.9631 & & \\
\hline $\mathrm{CD} 45 \mathrm{RO}+$ & $1.03(0.98-1.07)$ & 0.2625 & & \\
\hline $\mathrm{CD} 45 \mathrm{RA}+/ \mathrm{CD} 45 \mathrm{RO}+$ & $1.05(0.91-1.21)$ & 0.5254 & & \\
\hline CD56+ & $1.01(0.98-1.04)$ & 0.5395 & & \\
\hline Neutrophils/lymphocytes & $1.02(0.96-1.08)$ & 0.4844 & & \\
\hline EBV DNA & $1(1-1)$ & 0.3799 & & \\
\hline Cholinesterase & $1(1-1)$ & 0.1324 & & \\
\hline $\mathrm{Fb}$ & $1.88(1.21-2.9)$ & $0.0046^{* *}$ & $1.93(1.17-3.17)$ & $0.0098^{* *}$ \\
\hline
\end{tabular}

Note: ${ }^{*} P<0.05 ;{ }^{* *} P<0.01$; and ${ }^{* * *} P<0.001$.

TABLE 3: The critical value of related factors with radiation-induced hypothyroidism.

\begin{tabular}{|c|c|c|c|c|c|}
\hline \multirow{2}{*}{ Variables } & & \multicolumn{2}{|c|}{ Hypothyroidism } & \multirow{2}{*}{$\chi^{2}$} & \multirow{2}{*}{$P$ value } \\
\hline & & No & Yes & & \\
\hline \multirow{2}{*}{ TPO-Ab (IU/mL) } & $\leq 7.40$ & 24 & 3 & 15.1364 & $<0.001^{* * *}$ \\
\hline & $>7.40$ & 69 & 74 & & \\
\hline \multirow{2}{*}{ TG-Ab (IU/mL) } & $\leq 57.40$ & 81 & 48 & 14.1097 & $<0.001^{* * *}$ \\
\hline & $>57.40$ & 12 & 29 & & \\
\hline \multirow{2}{*}{$\mathrm{TG}(\mathrm{ng} / \mathrm{mL})$} & $\leq 16.65$ & 82 & 50 & 13.1046 & $<0.001^{* * *}$ \\
\hline & $>16.65$ & 11 & 27 & & \\
\hline \multirow{2}{*}{$\mathrm{Fb}(\mathrm{g} / \mathrm{L})$} & $\leq 3.50$ & 68 & 39 & 9.1172 & $0.003^{* *}$ \\
\hline & $>3.50$ & 25 & 38 & & \\
\hline
\end{tabular}

Note: ${ }^{* *} P<0.01 ;{ }^{* * *} P<0.001$.

dose limit of the thyroid gland, several studies have explored this topic, while ignoring the internal relationship between multiple systems in the body with hypothyroidism, which is an endocrine disease.
4.1. Thyroid-Associated Antibodies Associated with Hypothyroidism. The most common cause of hypothyroidism is autoimmune disease, and a large number of studies have shown that TPO-Ab and TG-Ab are closely related to 


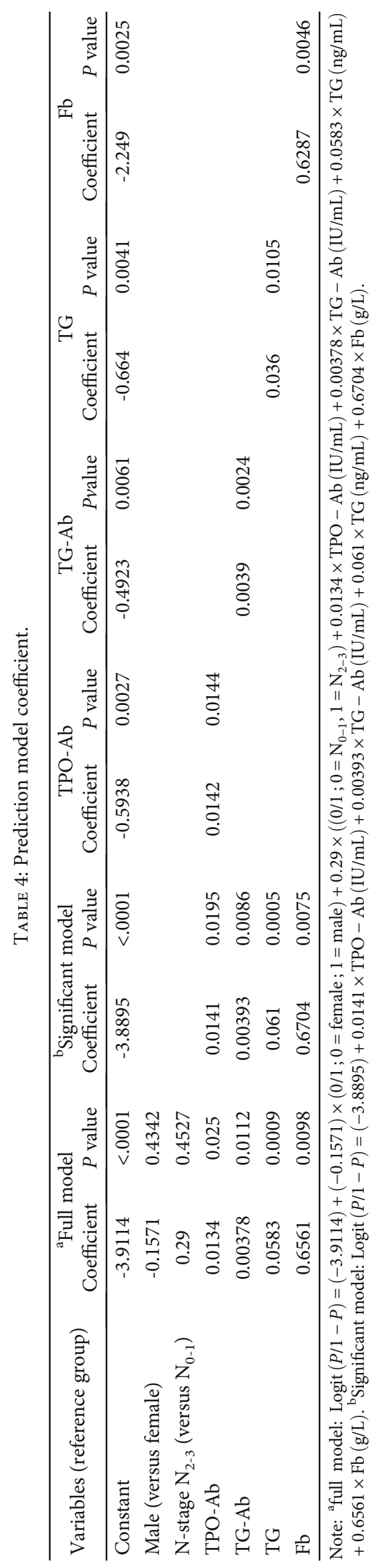




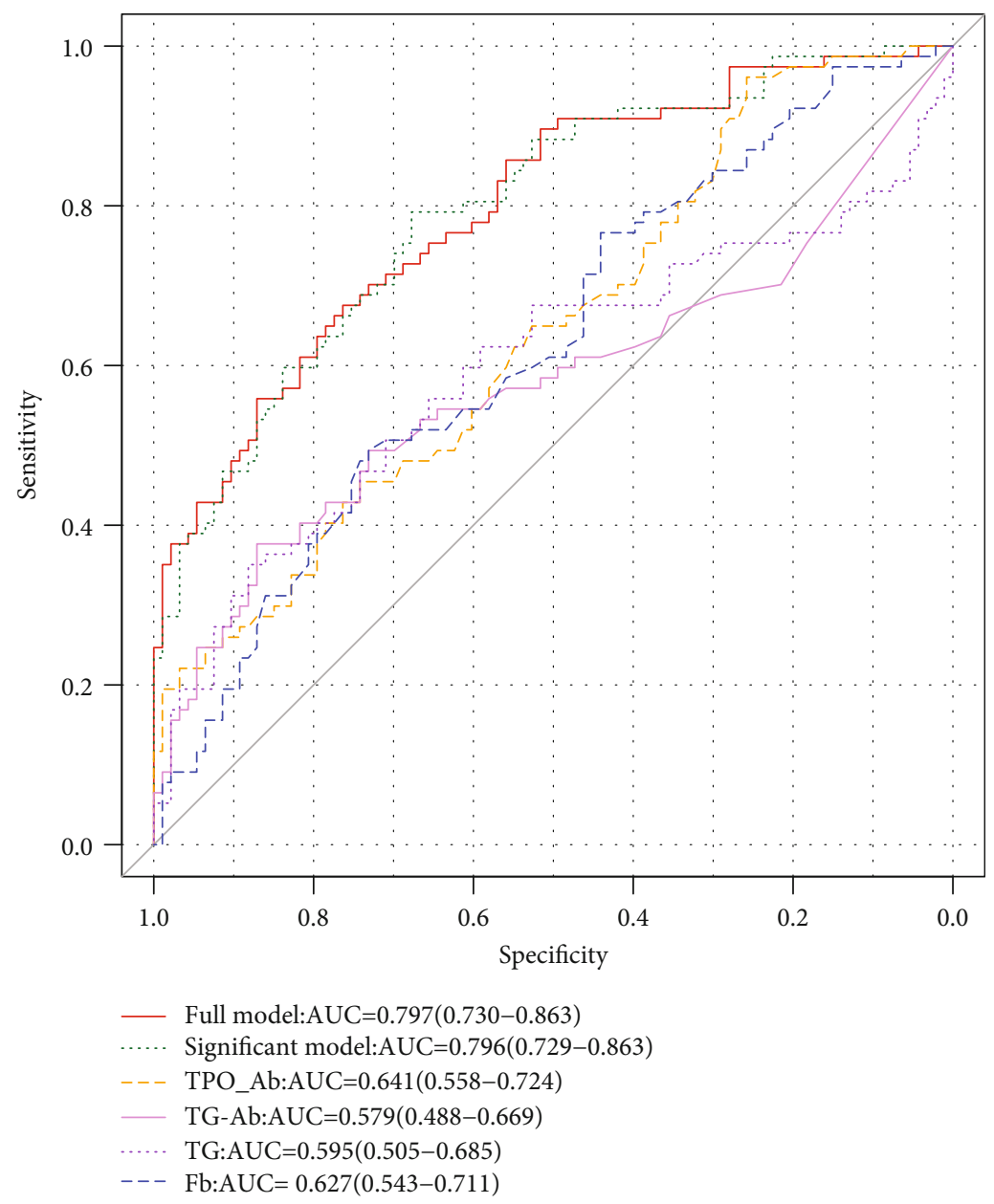

FIGURE 1: Analysis of the area under the ROC curve in patients with NPC treated with IMRT.

hypothyroidism [13]. Moreover, patients positive for TPO$\mathrm{Ab}$ and TG-Ab are more likely to develop hypothyroidism [23], and their concentrations can predict whether subclinical hypothyroidism will develop into clinical hypothyroidism [14]. This is consistent with our findings which showed that $\mathrm{TG}$, TG-Ab, and TPO-Ab are independent predictors of hypothyroidism. In addition, TG is primarily synthesized and secreted by thyroid follicular cells and regulated by TSH. As an autoantibody produced by TG, the higher the level of TG-Ab expression, the more serious the immune function disorder [24]. TPO is not only a key enzyme involved in thyroid hormone synthesis but also an important antigen responsible for causing autoimmune thyroid disease. Moreover, the abnormal expression of TPO and the immune response associated with TPO-Ab are important mechanisms of thyroid cell injury in patients with hypothyroidism. After radiotherapy, thyroid inflammation occurs in patients with nasopharyngeal carcinoma, which results in increased TG-Ab and TPO-Ab expression. Such expression impacts the synthesis of thyroid hormones and leads to hypothyroidism. Based on the information published in previous studies, we found that the increased expressions of TG, TG-Ab, and $\mathrm{TPO}-\mathrm{Ab}$ in the serum are important predictors of hypothyroidism. In addition, these predictors are also combined with all independent predictors to establish a model for predicting the incidence of hypothyroidism with a favorable prediction efficiency. Moreover, cross-validation was performed internally, which can be just as effective as using a separate test set to estimate the generalization error of the training set.

Although several studies have shown that hypothyroidism is related to TPO-Ab, TG- $\mathrm{Ab}$, and TG, few studies have investigated the relationship between radiation-induced hypothyroidism and thyroid-associated antibodies. Lin et al. [25] believed that there was a strong correlation between the levels of thyroid hormones and thyroidassociated antibodies in patients with NPC after radiotherapy; however, they did not further explore the level of thyroid-associated antibody expression related to hypothyroidism. Our study is the first to explore the correlation between NPC, radiotherapy, immunity, and hypothyroidism. The level of thyroid-associated antibody expression, immune cells, and coagulation function indexes before radiotherapy were used to screen high-risk patients with hypothyroidism. We found that the level of thyroid-associated antibody expression and fibrinogen were independent predictors of radiation-induced hypothyroidism. It was additionally shown that the significant model combined with TPO-Ab, TG-Ab, TG, and $\mathrm{Fb}$ was the optimal method used to predict the incidence of hypothyroidism after radiotherapy. The model was internally verified to exhibit a good performance 


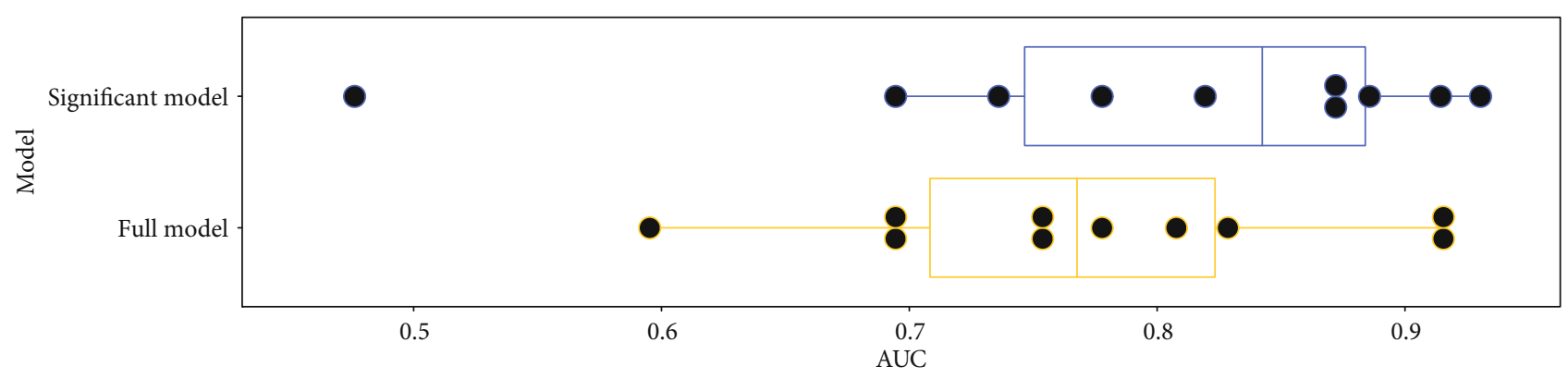

(a)

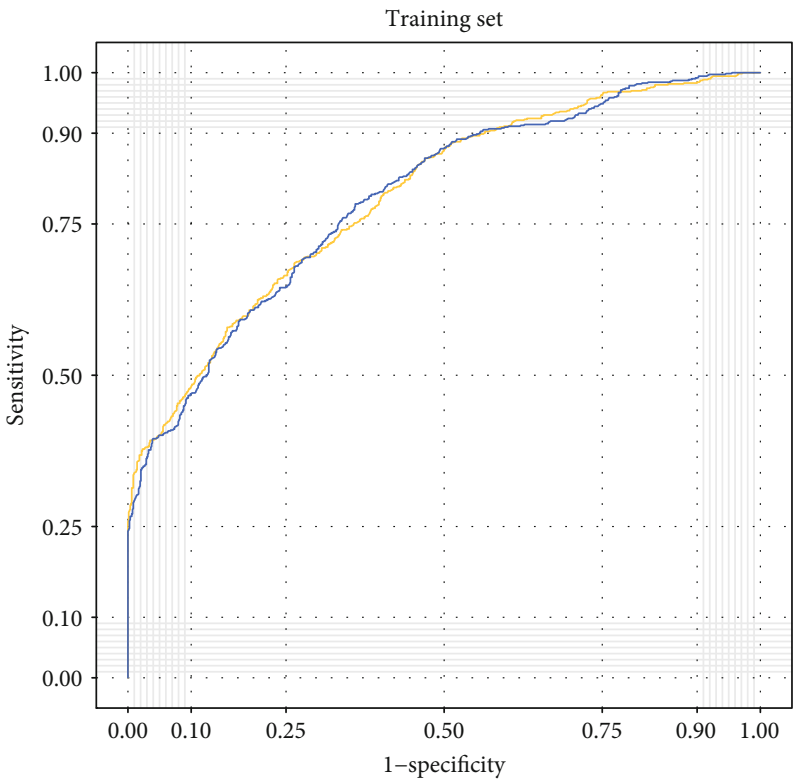

Full model

- Significant model

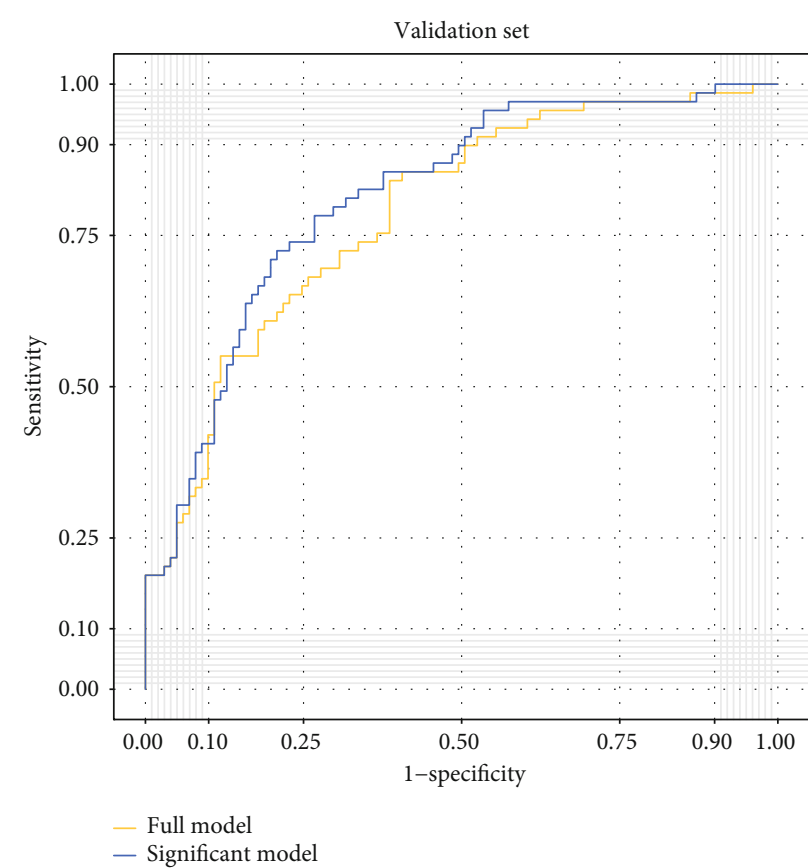

(c)

Figure 2: Predictive power comparison between the full model and significant model under multivariate analysis. (a) Box plot of AUC distribution of the 10 logistic regression models in each tenfold cross-validation. (b) Training ROC curves for the full model (red) and significant model (blue). The AUCs of the two curves are 0.794 and 0.792 , respectively. (c) Testing ROC curves for the full model (red) and significant model (blue). The AUCs of the two curves are 0.774 and 0.798 , respectively.

using the tenfold cross-validation method. The cut-off of $\mathrm{TPO}-\mathrm{Ab}$ and TG-Ab for hypothyroidism after radiotherapy is $7.40 \mathrm{IU} / \mathrm{mL}$ and $57.40 \mathrm{IU} / \mathrm{mL}$, respectively, which is much less than the lower limit of normal. Together, these findings suggest that immunity plays an important role in thyroid function damage caused by radiotherapy.

4.2. Immune Cells Associated with Hypothyroidism. Over the past century, there have been multiple studies on the humoral and cellular immune mechanisms related to autoimmune thyroid diseases, especially humoral immunity [26]. Segundo et al. [27] identified a class of B lymphocytes (IgM-, IgD-, CD44-, CD38++, CD71+, and CD95+) in the thyroid gland that was closely related to the concentration of TPO-Ab in the serum. Moreover, the CD4+ to CD8+ T cell ratio is also an important cause of autoimmune disease. The purpose of this study was to analyze the relationship between $\mathrm{T}$ cell subsets $(\mathrm{CD} 3+, \mathrm{CD} 4+, \mathrm{CD} 8+$, and $\mathrm{CD} 4$ $+/ \mathrm{CD} 8+)$, B cells (CD19+), and NK cells (CD56+) with radiation-induced hypothyroidism. No differences were identified among the patients with and without hypothyroidism, which may be due to the complex relationship between radiotherapy, immunity, and thyroid function that is disturbed by other factors. Since this study was the first to explore the correlation between radiation hypothyroidism and immune function, future research should eliminate some interference factors and explore the mechanism between radiation hypothyroidism and immune function in greater depth.

4.3. Fb Associated with Hypothyroidism. Previous studies have shown that patients with hypothyroidism often have risk factors for cardiovascular disease (e.g., hypertension, high cholesterol, and hyperlipidemia) $[28,29]$. We found that the fibrinogen content in high-risk patients with hypothyroidism was significantly higher than that of normal patients. Moreover, fibrinogen is an important factor in atherosclerosis and thrombosis. In this study, most patients with 
hypothyroidism also had high levels of fibrinogen, exhibiting hypercoagulable blood. This is consistent with the results reported by Ordookhani and Burman [30], who considered the increased coagulation factor VII and plasminogen activator inhibitor-1 activity to be one of the main mechanisms leading to the hypercoagulable state in patients with hypothyroidism.

4.4. Other Factors Associated with Hypothyroidism. The neutrophil-to-lymphocyte ratio in the serum is a common prognostic indicator, which is often used to predict the therapeutic toxicity, systemic inflammation, and tissue damage of various cancers [31-33]. In this study, we also aimed to explore the correlation between the neutrophil-to-lymphoid ratio and radiation-induced hypothyroidism. However, the neutrophil-to-lymphoid ratio did not appear to predict the incidence of hypothyroidism in patients with NPC after radiotherapy.

The purpose of this study was to screen outpatients at a high risk of hypothyroidism who were sensitive to radiotherapy in an effort to prompt clinicians to closely monitor the thyroid function of high-risk patients after radiotherapy. Although interfering factors were excluded as much as possible, there is an inevitability of confounding factors associated with the retrospective analysis. There are some shortcomings associated with our study, including the retrospective nature and the median follow-up time of only 20 months. This may also be the reason why no central hypothyroidism was observed in this study. In the future, further prospective studies should be performed to explore the relationship between radiotherapy, immunity, and hypothyroidism in patients with NPC.

\section{Conclusions}

This study is the first to explore the relationship between hematological indexes (e.g., thyroid-associated antibodies, immune cells, coagulation function, and radiation-induced hypothyroidism) in patients with NPC. TPO-Ab, TG-Ab, $\mathrm{TG}$, and $\mathrm{Fb}$ were found to represent independent predictors of radiation hypothyroidism. Thus, a significant model combined with TPO-Ab, TG-Ab, TG, and $\mathrm{Fb}$ may be used to screen high-risk populations for hypothyroidism after radiotherapy $(\mathrm{AUC}=0.796)$.

\section{Data Availability}

The datasets supporting the conclusions of this article are included within the article.

\section{Ethical Approval}

This study was approved by the Medical Ethics Committee of Zhejiang Cancer Hospital with committee's reference number: IRB-2019-87.

\section{Conflicts of Interest}

The authors declare that there are no competing interests.

\section{Acknowledgments}

This work was supported by the National Natural Science Foundation of China (81672971) and Key Technologies Research and Development Program (2017YFC0113201).

\section{References}

[1] J. C. Lin, J. S. Jan, C. Y. Hsu, W. M. Liang, R. S. Jiang, and W. Y. Wang, "Phase III study of concurrent chemoradiotherapy versus radiotherapy alone for advanced nasopharyngeal carcinoma: positive effect on overall and progression-free survival," Journal of Clinical Oncology, vol. 21, no. 4, pp. 631-637, 2003.

[2] W. B. Huang, J. Y. W. Chan, and D. L. Liu, "Human papillomavirus and World Health Organization type III nasopharyngeal carcinoma: multicenter study from an endemic area in Southern China," Cancer, vol. 124, no. 3, pp. 530-536, 2018.

[3] M. K. M. Kam, R. M. C. Chau, J. Suen, P. H. K. Choi, and P. M. L. Teo, "Intensity-modulated radiotherapy in nasopharyngeal carcinoma: dosimetric advantage over conventional plans and feasibility of dose escalation," International Journal of Radiation Oncology • Biology • Physics, vol. 56, no. 1, pp. 145-157, 2003.

[4] V. Lee, S. Y. Chan, C. W. Choi et al., "Dosimetric predictors of hypothyroidism after radical intensity-modulated radiation therapy for non-metastatic nasopharyngeal carcinoma," Clinical Oncology, vol. 28, no. 8, pp. e52-e60, 2016.

[5] L. J. McDowell, K. Rock, W. Xu et al., "Long-term late toxicity, quality of life, and emotional distress in patients with nasopharyngeal carcinoma treated with intensity modulated radiation therapy," International Journal of Radiation Oncology • Biology • Physics, vol. 102, no. 2, pp. 340-352, 2018.

[6] N. Rodondi, W. P. J. den Elzen, D. C. Bauer et al., "Subclinical hypothyroidism and the risk of coronary heart disease and mortality," JAMA, vol. 304, no. 12, pp. 1365-1374, 2010.

[7] K. Balázs, E. Kis, C. Badie et al., "Radiotherapy-induced changes in the systemic immune and inflammation parameters of head and neck cancer patients," Cancers, vol. 11, no. 9, p. 1324, 2019.

[8] S. Falcke, P. Rühle, L. Deloch, R. Fietkau, B. Frey, and U. Gaipl, "Clinically relevant radiation exposure differentially impacts forms of cell death in human cells of the innate and adaptive immune system," International Journal of Molecular Sciences, vol. 19, no. 11, p. 3574, 2018.

[9] T. B. Dar, R. M. Henson, and S. L. Shiao, "Targeting innate immunity to enhance the efficacy of radiation therapy," Frontiers in Immunology, vol. 9, p. 3077, 2019.

[10] H.-y. Chen, L. Xu, L.-f. Li, X.-x. Liu, J.-x. Gao, and Y.-r. Bai, "Inhibiting the $\mathrm{CD}^{+} \mathrm{T}$ cell infiltration in the tumor microenvironment after radiotherapy is an important mechanism of radioresistance," Scientific Reports, vol. 8, no. 1, article 11934, 2018.

[11] S. L. Hancock, I. R. McDougall, and L. S. Constine, "Thyroid abnormalities after therapeutic external radiation," International Journal of Radiation Oncology • Biology • Physics, vol. 31, no. 5, pp. 1165-1170, 1995.

[12] B. Cutuli, P. Quetin, J.-F. Rodier, P. Barakat, and J.-C. Grob, "Severe hypothyroidism after chemotherapy and locoregional irradiation for breast cancer," Radiotherapy and Oncology, vol. 57, no. 1, pp. 103-105, 2000. 
[13] L. Chaker, A. C. Bianco, J. Jonklaas, and R. P. Peeters, "Hypothyroidism," The Lancet, vol. 390, no. 10101, pp. 1550-1562, 2017.

[14] G. Effraimidis, T. G. A. Strieder, J. G. P. Tijssen, and W. M. Wiersinga, "Natural history of the transition from euthyroidism to overt autoimmune hypo- or hyperthyroidism: a prospective study," European Journal of Endocrinology, vol. 164, no. 1, pp. 107-113, 2011.

[15] N. Y. Lee, Q. Zhang, D. G. Pfister et al., "Addition of bevacizumab to standard chemoradiation for locoregionally advanced nasopharyngeal carcinoma (RTOG 0615): a phase 2 multiinstitutional trial," The Lancet Oncology, vol. 13, no. 2, pp. 172-180, 2012.

[16] N. Bhandare, L. Kennedy, R. S. Malyapa, C. G. Morris, and W. M. Mendenhall, "Primary and central hypothyroidism after radiotherapy for head-and-neck tumors," International Journal of Radiation Oncology - Biology • Physics, vol. 68, no. 4, pp. 1131-1139, 2007.

[17] J. Beynon, S. Akhtar, and T. Kearney, "Predictors of outcome in myxoedema coma," Critical Care, vol. 12, no. 1, p. 111, 2008.

[18] M. I. Surks, E. Ortiz, G. H. Daniels et al., "Subclinical thyroid disease: scientific review and guidelines for diagnosis and management," JAMA, vol. 291, no. 2, pp. 228-238, 2004.

[19] A. Bielecka-Dabrowa, B. Godoy, T. Suzuki, M. Banach, and S. von Haehling, "Subclinical hypothyroidism and the development of heart failure: an overview of risk and effects on cardiac function," Clinical Research in Cardiology, vol. 108, no. 3, pp. 225-233, 2019.

[20] K. Sommat, W. S. Ong, A. Hussain et al., "Thyroid V40 predicts primary hypothyroidism after intensity modulated radiation therapy for nasopharyngeal carcinoma," International Journal of Radiation Oncology • Biology • Physics, vol. 98, no. 3, pp. 574-580, 2017.

[21] S. Ling, A. D. Bhatt, N. V. Brown et al., "Correlative study of dose to thyroid and incidence of subsequent dysfunction after head and neck radiation," Head Neck, vol. 39, no. 3, pp. 548-554, 2017.

[22] R.-P. Zhai, F.-f. Kong, C.-r. du, C.-s. Hu, and H.-m. Ying, "Radiation-induced hypothyroidism after IMRT for nasopharyngeal carcinoma: clinical and dosimetric predictors in a prospective cohort study," Oral Oncology, vol. 68, pp. 44-49, 2017.

[23] B. Biondi and D. S. Cooper, "The clinical significance of subclinical thyroid dysfunction," Endocrine Reviews, vol. 29, no. 1, pp. 76-131, 2008.

[24] T. H. Collet, D. C. Bauer, A. R. Cappola et al., "Thyroid antibody status, subclinical hypothyroidism, and the risk of coronary heart disease: an individual participant data analysis," The Journal of Clinical Endocrinology \& Metabolism, vol. 99, no. 9, pp. 3353-3362, 2014.

[25] Z. Lin, L. Chen, Y. Fang, A. Cai, T. Zhang, and V. W. C. Wu, "Longitudinal study on the correlations of thyroid antibody and thyroid hormone levels after radiotherapy in patients with nasopharyngeal carcinoma with radiation-induced hypothyroidism," Head Neck, vol. 36, no. 2, pp. 171-175, 2014.

[26] C. R. Strakosch, B. E. Wenzel, V. V. Row, and R. Volpe, "Immunology of autoimmune thyroid diseases," The New England Journal of Medicine, vol. 307, no. 24, pp. 1499-1507, 1982.

[27] C. Segundo, C. Rodríguez, M. Aguilar et al., "Differences in thyroid-infiltrating B lymphocytes in patients with Graves' disease: relationship to autoantibody detection," Thyroid, vol. 14, no. 5, pp. 337-344, 2004.
[28] D. Tiller, T. Ittermann, K. H. Greiser et al., "Association of serum thyrotropin with anthropometric markers of obesity in the general population," Thyroid, vol. 26, no. 9, pp. 12051214, 2016.

[29] C. Floriani, B. Gencer, T. H. Collet, and N. Rodondi, "Subclinical thyroid dysfunction and cardiovascular diseases: 2016 update," European Heart Journal, vol. 39, no. 7, pp. 503-507, 2018.

[30] A. Ordookhani and K. D. Burman, "Hemostasis in hypothyroidism and autoimmune thyroid disorders," International Journal of Endocrinology and Metabolism, vol. 15, no. 2, article e42649, 2017.

[31] L. Wu and Y. L. Chung, "Tumor-infiltrating T cell receptorbeta repertoires are linked to the risk of late chemoradiationinduced temporal lobe necrosis in locally advanced nasopharyngeal carcinoma," International Journal of Radiation Oncology • Biology • Physics, vol. 104, no. 1, pp. 165-176, 2019.

[32] Z. Mei, L. Shi, B. Wang et al., "Prognostic role of pretreatment blood neutrophil-to-lymphocyte ratio in advanced cancer survivors: a systematic review and meta-analysis of 66 cohort studies," Cancer Treatment Reviews, vol. 58, pp. 1-13, 2017.

[33] C. R. Haddad, L. Guo, S. Clarke, A. Guminski, M. Back, and T. Eade, "Neutrophil-to-lymphocyte ratio in head and neck cancer," Journal of Medical Imaging and Radiation Oncology, vol. 59, no. 4, pp. 514-519, 2015. 